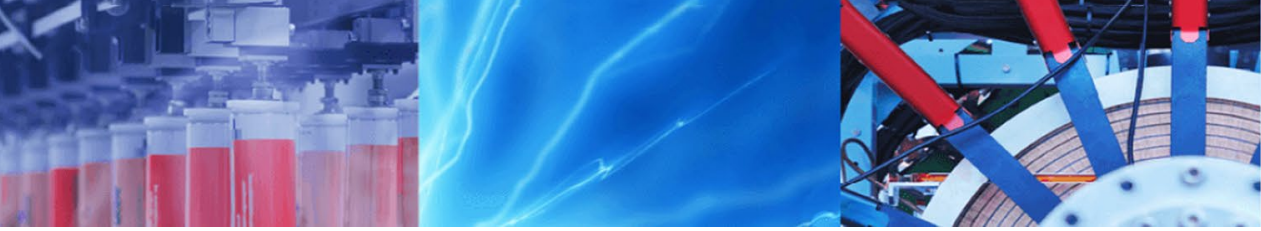

Research Article

\title{
Effect of chromium on structural, morphological and electrical properties of lithium ferrite nanoparticles
}

\author{
D. Ravinder Nayak ${ }^{1}$ K. Chandra Babu Naidu ${ }^{2}$ D. Ravinder ${ }^{1}$
}

(c) Springer Nature Switzerland AG 2019

\begin{abstract}
The $\mathrm{Li}_{0.5} \mathrm{Fe}_{2.5-\mathrm{x}} \mathrm{Cr}_{\mathrm{x}} \mathrm{O}_{4}(\mathrm{x}=0.0-1.0)$ nanoparticles were prepared by the citrate gel autocombustion method. The diffraction spectra were carried out in order to confirm the formation of the single phase cubic spinel structure. The lattice parameter was noted to be decreasing from 8.433 to $8.381 \AA$ with increase in $\mathrm{Cr}$-content from 0.0 to 1.0. The morphology was analyzed using scanning electron microscopy and transmission electron microscopy. The achieved results revealed that the particles were almost spherical in shape. Further, the dc-electrical conductivity $\left(\sigma_{\mathrm{dc}}\right)$ of nanoferrites was described as a function of composition and temperature. In addition, the dielectric properties such as dielectric constant $\left(\varepsilon^{\prime}\right)$ and dielectric loss ( $\tan \delta$ ) were also discussed as a function of frequency at various temperatures.
\end{abstract}

Keywords Nanoparticles · Chromium · Structural studies · X-ray · Electronic transfer

\section{Introduction}

It was a well familiar fact that the ferrites contain considerable significance towards the applications of magnetic memories, electronic devices, and high frequency devices because of their low dc electrical conductivity, magnetic coercivity, magnetic relative loss factor, eddy current loss, and dielectric loss [1-3]. Normally, in the case of lithium iron oxide $\left(\mathrm{Li}_{0.5} \mathrm{Fe}_{2.5} \mathrm{O}_{4}\right)$ inverse spinel structure, the lithium cations $\left(\mathrm{Li}^{+}\right)$will have the greater probability to prefer the octahedral (B) sites. On the other hand, the ferric ions $\left(\mathrm{Fe}^{3+}\right)$ give high preference to occupy the tetrahedral (A) sites within the $\mathrm{Li}_{0.5} \mathrm{Fe}_{2.5} \mathrm{O}_{4}$ structure $[4,5]$. However, the lithium ferrites (LF) and LFs based materials were specifically confined to potential microwave device applications viz., phase shifting devices, transformer and inductor core devices, isolators/circulators, antenna devices, electrode devices etc., due to the high magnetic Curie transition temperature $\left(T_{c}\right)$, low relative loss factor (rlf), small value of electrical conductivity, high saturation magnetization $\left(M_{s}\right)$, low loss, and other magnetic properties. Therefore, depending on the above mentioned properties, one can justify that the LF and LFs substituted nanomaterials can reveal several applications because of their better performance when compared with the other spinels [6-9].

However, it was an established fact that the synthesis approach can show a vital role in achieving the enhanced electrical, magnetic and optical properties of ferrites in both bulk as well as nanoform. There were several methods in order to synthesize ferrites viz., microwave hydrothermal, flash combustion, co-precipitation, sol-gel, and citrate gel methods [10]. Upon making a comparison of all these synthesis techniques, citrate gel auto combustion technique showed more attraction owing to the usage of low operating temperatures, less time consumption for the completion of reaction, homogenous mixture of material, low (nano) sized particles etc. In this concern, previous scientists investigated various electrical and magnetic properties of the lithium cadmium [11], lithium magnesium [13] and lithium zinc [12]. Moreover, many researchers prepared the bulk and nano lithium chromium ferrites [13-23] for various electrical, dielectric, magnetic,

D. Ravinder, ravindergupta28@rediffmail.com | 'Department of Physics, Osmania University, Hyderabad, Telangana 500007, India. ${ }^{2}$ Department of Physics, GITAM Deemed to be University, Bangalore, Karnataka 562163, India.

SN Applied Sciences (2019) 1:235 | https://doi.org/10.1007/s42452-019-0258-1 
thermal, spectroscopic, photo acoustic and optical properties. But very few reports were found in the literature on dc-electrical conductivity, ac-electrical conductivity, and dielectric properties of $\mathrm{Li}-\mathrm{Cr}$ nanoferrites synthesized via the citrate gel autocombustion technique. Hence, the authors interested to report the above said properties for the present materials.

\section{Experimental details}

\subsection{Materials}

In order to synthesis the $\mathrm{LiCr}$ ferrite nanoparticles, the raw materials were selected as the Lithium nitrate$\mathrm{Li}\left(\mathrm{NO}_{3}\right)$ (sigma Aldrich $99 \%$ pure AR grade), Ferric Nitrate$\left(\mathrm{Fe}\left(\mathrm{NO}_{3}\right)_{2} \cdot 9 \mathrm{H}_{2} \mathrm{O}\right)$ (98\% pure GR grade), Chromium Nitrate$\left(\mathrm{Cr}\left(\mathrm{NO}_{3}\right)_{2} \cdot 9 \mathrm{H}_{2} \mathrm{O}\right.$ ) (Otto Chemie Pvt. Limited, $98 \%$ pure GR grade), Citric acid- $\left(\mathrm{C}_{6} \mathrm{H}_{8} \mathrm{O}_{7} \cdot \mathrm{H}_{2} \mathrm{O}\right)$ (SDFCL-SDFINE Chem. Limited, 99\% pure AR grade), and Ammonia-( $\left.\mathrm{NH}_{3}\right)$ (SDFCLSDFINE Chem. Limited, 99\% pure AR grade).

\subsection{Synthesis method}

In order to synthesize the chromium substituted lithium ferrites nanoparticles, the above mentioned chemicals were selected. Further, these were weighed as per the prepared stoichiometric ratio of metal nitrates. These chemicals were dissolved in distilled water as well. Afterwards, the nitrate materials and citric acid were mixed together according to the molar ratio to perform the citrate gel auto-combustion method. Herein, the nitrate materials worked as oxidizing agents while organic fuels acted as reducing agents. Latter, different material properties were sequentially altered by changing the ratios of oxidant to fuel samples. In such a way, the individual solutions were prepared as well and furthermore mixed by following the stirring about $1 \mathrm{~h}$ on a magnetic stirrer containing hot plate. In addition, the dilute $\mathrm{NH}_{3}$ solution was added slowly in order to maintain the $\mathrm{pH}$ value equals to 7 . Now the viscous gel was formed. Subsequently, the hot plate temperature was maintained at $80^{\circ} \mathrm{C}$. As a result of this, the dry gel was formed. This fragment was again subjected to autocombustion process to produce the ferrite powder particles. After completion of this, the powder sample was grinded as well and further characterized using various characterization techniques such as X-ray diffraction, Scanning and transmission electron microscopy, two probe measurement, and LCR controller for structural, morphological, dc electrical conductivity and ac electrical properties respectively. The flow chart representing the synthesis method of present ferrite materials was shown in Fig. 1.

\section{Results and discussion}

\subsection{X-ray diffraction pattern}

The X-ray diffraction (XRD) patterns of $\mathrm{Cr}$-substituted lithium nanoferrites were shown in Fig. 2. It can be
Fig. 1 Synthesis flow chart of Li-Cr nano ferrites

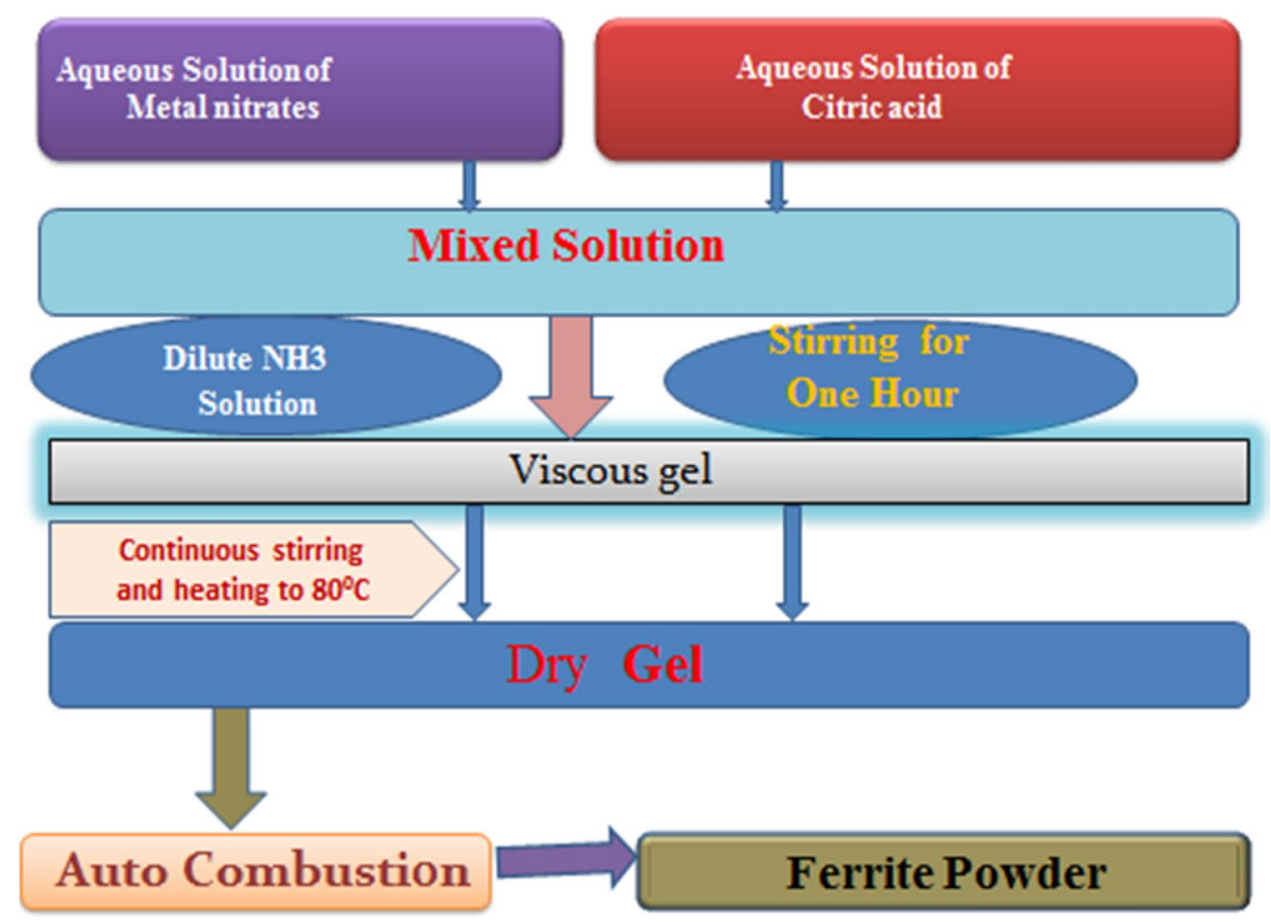


Fig. 2 XRD patterns of nanocrystalline $\mathrm{Li}-\mathrm{Cr}$ ferrites

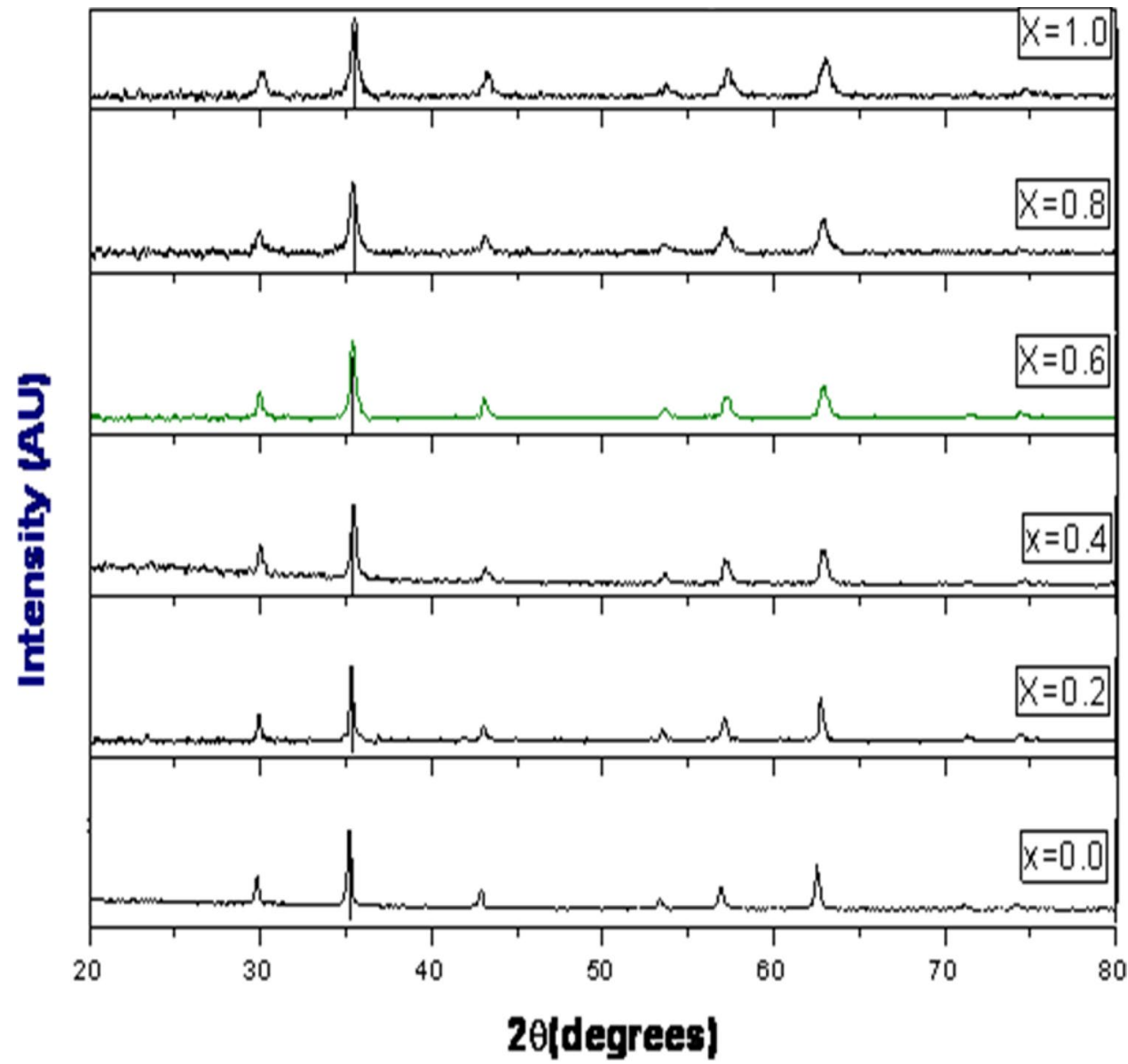

Table 1 Structural parameters of nanocrystalline Li-Cr ferrites

\begin{tabular}{lllll}
\hline$x$ & MW (gm/mole) & $\begin{array}{l}\text { Crystallite size } \\
(\mathrm{nm}) \pm 0.2 \mathrm{~nm}\end{array}$ & $\begin{array}{l}\text { Lattice } \\
\text { parameter } \\
(\AA)\end{array}$ & $\begin{array}{l}\text { X-ray density } \mathrm{d}_{\mathrm{x}} \\
\left(\mathrm{g} / \mathrm{cm}^{3}\right) \pm 0.02\end{array}$ \\
\hline 0.0 & 207.091 & 27 & 8.433 & 4.760 \\
0.2 & 206.321 & 21 & 8.414 & 4.600 \\
0.4 & 205.551 & 26 & 8.401 & 4.611 \\
0.6 & 204.781 & 21 & 8.400 & 4.587 \\
0.8 & 204.001 & 21 & 8.397 & 4.575 \\
1.0 & 203.241 & 17 & 8.381 & 4.565 \\
\hline
\end{tabular}

obviously understood that the diffraction pattern of the prepared samples confirmed the formation of welldefined single phase cubic spinel structure. The average crystallite size of the prepared nanoferrites measured from the full width half maxima of high intense peak (311) and was found to be in the range 17-27 nm by using Scherrer formula [4]. The XRD analysis of the prepared $\mathrm{Li}-\mathrm{Cr}$ nanocrystalline samples provided the estimation of the lattice constant (a), average crystallite size (D), X-ray density $\left(d_{x}\right)$ etc., as given in Table 1 . The lattice parameter was noted to be decreasing from 8.433 to $8.381 \AA$ with increase in $\mathrm{Cr}$-content from 0.0 to 1.0. It was occurred due to the occupation of $\mathrm{Fe}^{+3}(0.645 \AA)$ ions of larger ionic radii by the $\mathrm{Cr}^{+3}(0.615 \AA)$ ions of smaller ionic radii [23]. In addition, it was a known fact that once the $\mathrm{Cr}^{+3}$ ions were doped to the lithium ferrite, the spinel lithium ferrite started to shrink. Thus, it can induce uniform strain in the lattice as the specimen elastically deformed. This can in turn lead to the lattice interplanar spacing to change the position of diffraction peak. Thus, it caused to decrease of lattice constant with increase in $\mathrm{Cr}$-content [23]. The X-ray density was observed to be decreasing from 4.760 to $4.565 \mathrm{~g} / \mathrm{cm}^{3}$ with increase of $\mathrm{Cr}$-content. It can be attributed to the decrease of molecular weight (MW) from 207 to $203 \mathrm{~g} /$ mole with dopant. Moreover, it can be seen from the Table 2 that the Curie temperature was decreased with increase in chromium content. This established a fact that the addition of chromium in lithium ferrite system showed the loss in densification. This kind of result was also observed by the earlier researchers [24] in the case of $\mathrm{Mn}-\mathrm{Zn}$ ferrites and Li-Zn ferrites [25]. 
Table 2 Electrical resistivity and activation energies of the $\mathrm{Li}_{0.5} \mathrm{Fe}_{2.5-\mathrm{x}} \mathrm{Cr}_{\mathrm{x}} \mathrm{O}_{4}$ system

\begin{tabular}{|c|c|c|c|}
\hline \multirow[t]{2}{*}{ Composition } & \multirow{2}{*}{$\begin{array}{l}\text { Curie tempera- } \\
\text { ture }\left({ }^{\circ} \mathrm{C}\right)\end{array}$} & \multicolumn{2}{|c|}{ Activation energy (eV) } \\
\hline & & $\begin{array}{l}\text { Ferrimagnetic } \\
\text { region }\end{array}$ & $\begin{array}{l}\text { Para- } \\
\text { magnetic } \\
\text { region }\end{array}$ \\
\hline $\mathrm{Li}_{0.5} \mathrm{Fe}_{2.5} \mathrm{O}_{4}$ & $>600$ & 1.34 & - \\
\hline $\mathrm{Li}_{0.4} \mathrm{Cr}_{0.2} \mathrm{Fe}_{2.4} \mathrm{O}_{4}$ & 572 & 0.875 & 0.945 \\
\hline $\mathrm{Li}_{0.3} \mathrm{Cr}_{0.4} \mathrm{Fe}_{2.3} \mathrm{O}_{4}$ & 560 & 0.703 & 0.798 \\
\hline $\mathrm{Li}_{0.2} \mathrm{Cr}_{0.6} \mathrm{Fe}_{2.2} \mathrm{O}_{4}$ & 553 & 0.526 & 0.659 \\
\hline $\mathrm{Li}_{0.1} \mathrm{Cr}_{0.8} \mathrm{Fe}_{2.1} \mathrm{O}_{4}$ & 540 & 0.480 & 0.621 \\
\hline $\mathrm{CrFe}_{2} \mathrm{O}_{4}$ & 520 & 0.575 & 0.712 \\
\hline
\end{tabular}

\subsection{Surface morphology}

The FESEM and TEM were used to examine the topographic characteristics of the synthesized samples. The FESEM micrographs of present samples were shown in Fig. 3. At $x=0$, well defined spherical grains were noticed. On the other hand, in case of $x=0.2-1.0$, clustered like grains were found. In general, this kind of nature may be inferred due to the high agglomeration among the nanosized grains. The grain size was observed to be varying from 85 to $192 \mathrm{~nm}$ for all the $\mathrm{Cr}$-contents.

In addition, the TEM pictures of the same samples were presented in Fig. 4. The lithium ferrite revealed almost spheres like nanoparticles. Upon adding the $\mathrm{Cr}$-element in the lithium ferrite system, the well agglomerated nanoparticles were formed. In other words, one can treat it as piling up behavior of nanoparticles on top of each other. The magnetic interactions among nanoparticles were responsible for the observed agglomeration behavior [26-30]. Latter, the average particle size was found to be of order 18-43 $\mathrm{nm}$. This established a fact that there was a huge variation between grain size and particle size. It can be normally acquired as a result of agglomeration of nanoparticles. Moreover, the selected area electron diffraction pattern (SAED) of $x=0.6$ was shown in Fig. 5 . It exhibited the formation of all single phase cubic spinel reflections which in turn supported the $x$-ray diffraction pattern of the current samples. Besides, the SAED confirmed the well crystalline nature of prepared ferrite nanoparticles based on the superposition of the bright spot with Debye ring pattern.

\subsection{DC resistivity studies}

DC electrical resistivity $\left(\rho_{D C}\right)$ measurement is a powerful method in order to elucidate the electrical conductivity mechanism. The $\rho_{D C}$ of the $\mathrm{Cr}$-substituted lithium ferrite nanomaterials was calculated using the two probe technique. The powder samples were prepared in the form of pellets and further all the pellets were sputtered with silver paste to work as ohmic contact. The pellets were inserted between the electrodes of the sample holder to find the DC-resistivity. With the help of $\rho_{D C}$, the DC-electrical conductivity was computed and therefore the conduction mechanism can be well understood. It was a well known fact that the electrical conduction mechanism in ferrites was mostly as a result of hopping of charge carriers (electrons). It can depend mainly on the distance between two cations [31]. Therefore, the there may exist the electrostatic interactions in the case of cations and the charge carriers. Latter, it led to enhancement of polarization in the nearby portions of the cations. Hence, one can expect that the charge carriers will have the possibility to locate at the centre of the polarization. In next step, these charge carriers can be migrated to the nearest lattice sites as a result of internal thermal energy. Therefore, this type of mechanism in conduction can be called hopping mechanism [32].

The two probe method can provide the voltage and current parameters and further the $D C$ resistivity of the sample was calculated by using the relation: $\rho_{D C}=R A / I$ $(\Omega-\mathrm{cm})$, herein R: sample resistance, A: area of cross section pellet $=3.1416 \mathrm{r}^{2}$, : sample radius and $\mathrm{I}$ : the thickness of pellet. In the case of $L F$, the electrical conduction mechanism can be observed between the ferrous and ferric ions within the ferrite structure. That is, $\mathrm{Fe}^{+2} \leftrightarrow \mathrm{Fe}^{+3}+\mathrm{e}^{-}$. The charge transfer happened between the adjacent octahedral sites. In addition, this kind of local displacement of electrons was responsible for the polarization the ferrite specimen [33]. In this work, the dc electrical conductivity $\left(\sigma_{D C}\right)$ behavior of each sample was studied as function of temperature ranging from 200 to $600{ }^{\circ} \mathrm{C}$. It expressed that the $\sigma_{D C}$ of $C r$ substituted LF materials was increased upon increasing the temperature and thus, it indicated the magnetic semiconducting nature of these materials. The general form of DC-electrical conductivity of the materials was mathematically given by $\sigma_{D C} \sigma_{o} \exp \left(-E_{a} / K_{b} T\right)$, where $E_{a}$ is the thermal activation energy, $\sigma_{o}$ is the pre-exponential factor depending on nature of the material and $K_{b}$ is the Boltzmann constant [34].

The variation of DC-electrical conductivity of present nanoparticles as a function of temperature was investigated by drawing graphs of Log ( $\sigma . T$ ) versus $10^{3} / \mathrm{T}$ as depicted in Fig. 6. All the plots of nanomaterials performed almost the straight line behavior by maintaining certain slopes, wherein the slope values can give rise to the activation energies of the corresponding samples. It can be obviously understood from Fig. 6 that all the plots other than LF sample exhibited the variation in slope obtained from the electrical conductivity (log $\sigma . T$ ) versus $10^{3} / T$ plots at a particular temperature. We noted the slope change in the Arrhenius plots which may be commonly occurred as 

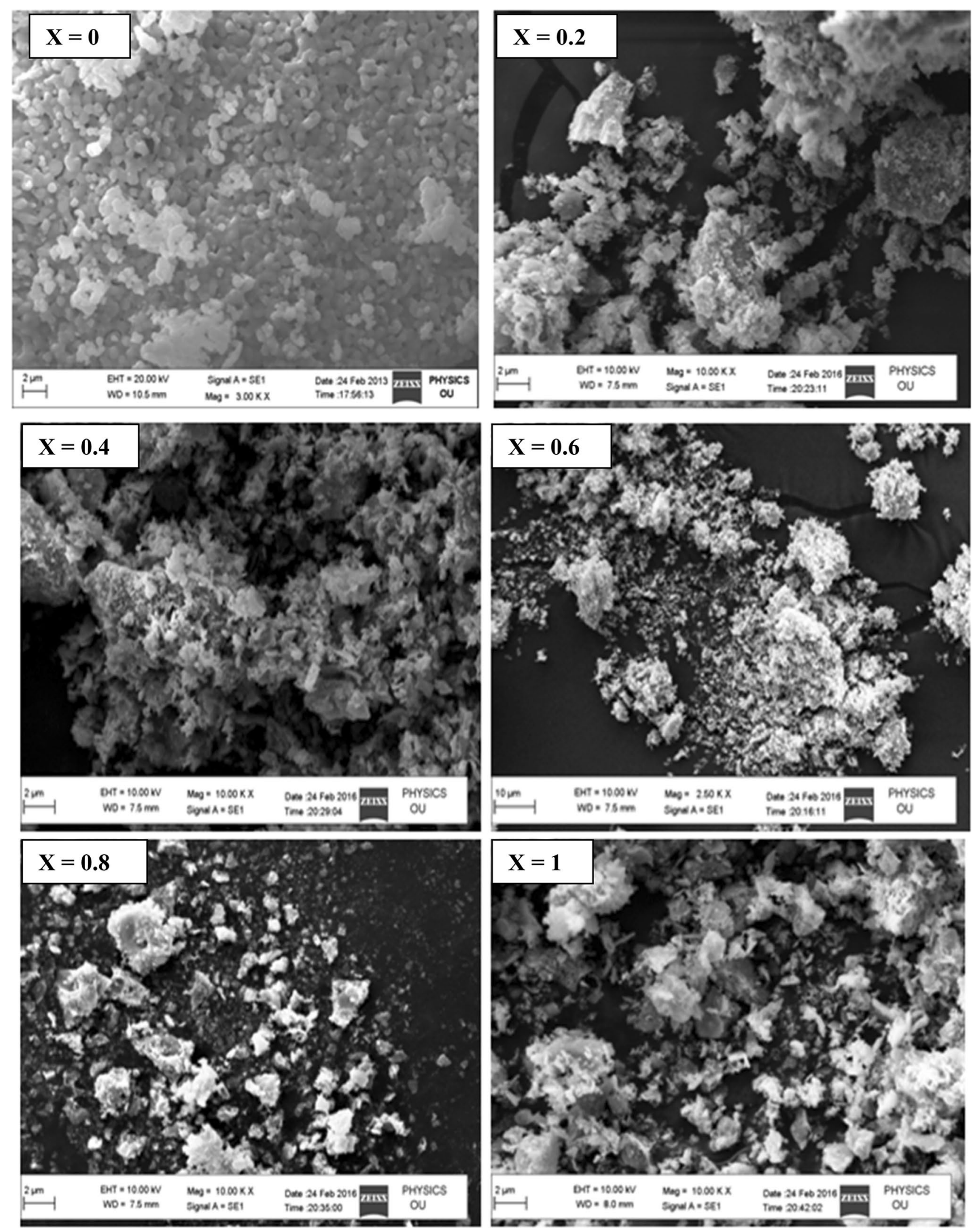

Fig. 3 FESEM micrographs of nanocrystalline Li-Cr ferrites 

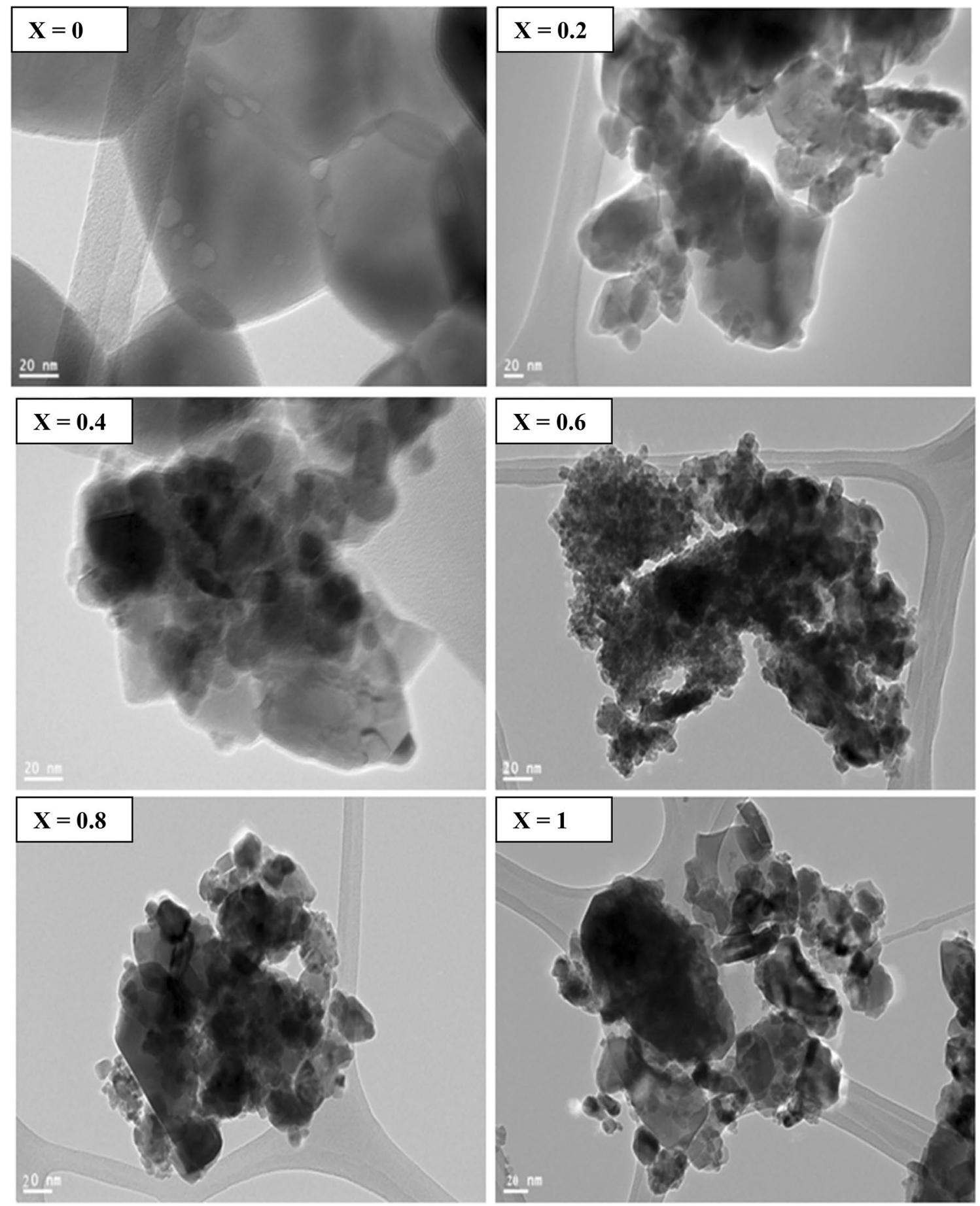

Fig. 4 TEM images of $\mathrm{Li}_{0.5} \mathrm{Fe}_{2.5-\mathrm{x}} \mathrm{Cr}_{\mathrm{x}} \mathrm{O}_{4}(\mathrm{X}=0.0$ to 1.0$)$

a result of the change of straight line at the Curie transition temperature $\left(T_{c}\right)$. Normally, this kind of manner was attained owing to the high randomization of magnetic spins. Furthermore, the ferri to paramagnetic transition took place at the $T_{c}$. But beyond $T_{c^{\prime}}$ the falling nature of magnetic spins took place and hence it indicated the paramagnetism behavior. Thus, the change of magnetic state was alone responsible for achieving two activation energies in low and high temperature regions. The obtained values of Curie temperature and dc activation energies of $\mathrm{Cr}$ substituted LF materials in ferri and paramagnetic regions were listed in Table 2 . From Table 2, it was clear that the magnetic Curie transition temperature was decreased from $>600$ to $520^{\circ} \mathrm{C}$ with increase of $\mathrm{Cr}^{+3}$ 


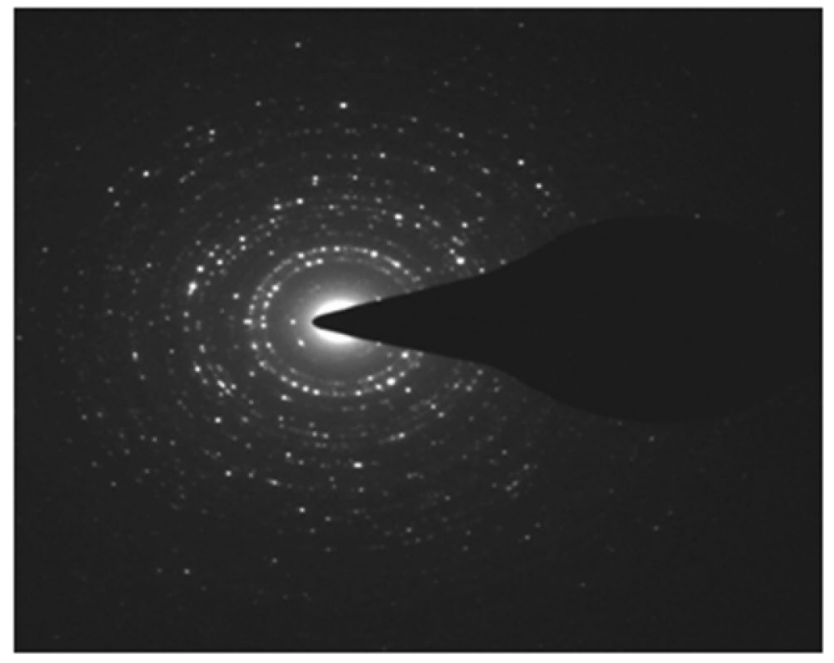

Fig. 5 SAED of $x=0.6$

cations in the lithium ferrite spinel structure. This kind of manner may be manifested due to the decrease of magnetic exchange interaction between the octahedral and tetrahedral sites [31]. In the X-ray diffraction spectral analysis, it was confirmed that the lattice constant was decreased with increase in $\mathrm{Cr}$-content. Therefore, the distance between the A and B-sites can be increased. As a result, the magnetic exchange $(A-B)$ interaction can be decreased between the two sites. In addition, the magnitudes of activation energies of present samples in the ferrimagnetic region were smaller than that of activation energies in paramagnetic region [32-36]. It can be achieved owing to the small value of electrical conductivity in paramagnetic region and high value of the same in ferrimagnetic region. However, this established a fact that the activation energy and electrical conductivity are reciprocal to each other.

\subsection{Dielectric properties}

The frequency dependence of dielectric constant $\left(\varepsilon^{\prime}\right)$ and dielectric loss (tan $\delta$ ) of Cr-substituted LF samples at four different temperatures (room temperature, $100{ }^{\circ} \mathrm{C}, 300^{\circ} \mathrm{C}$, and $500{ }^{\circ} \mathrm{C}$ ) was depicted in Fig. 7. The $\varepsilon^{\prime}$ versus frequency plot demonstrated that both $\varepsilon^{\prime}$ as well as $\tan \delta$ started diminishing with increase in frequency from $100 \mathrm{~Hz}$ to $5 \mathrm{MHz}$. It was a usual dielectric behavior of ceramic materials that the $\varepsilon^{\prime}$ is decreasing with increase of frequency for all samples. The Koop's phenomenon or double-layer dielectric model provided a theoretical elucidation for achieving larger values of $\varepsilon^{\prime}$ and $\tan \delta$ at smaller frequencies [34]. The doublelayer dielectric model suggested that the polycrystalline ceramic material can pertain two layers termed as
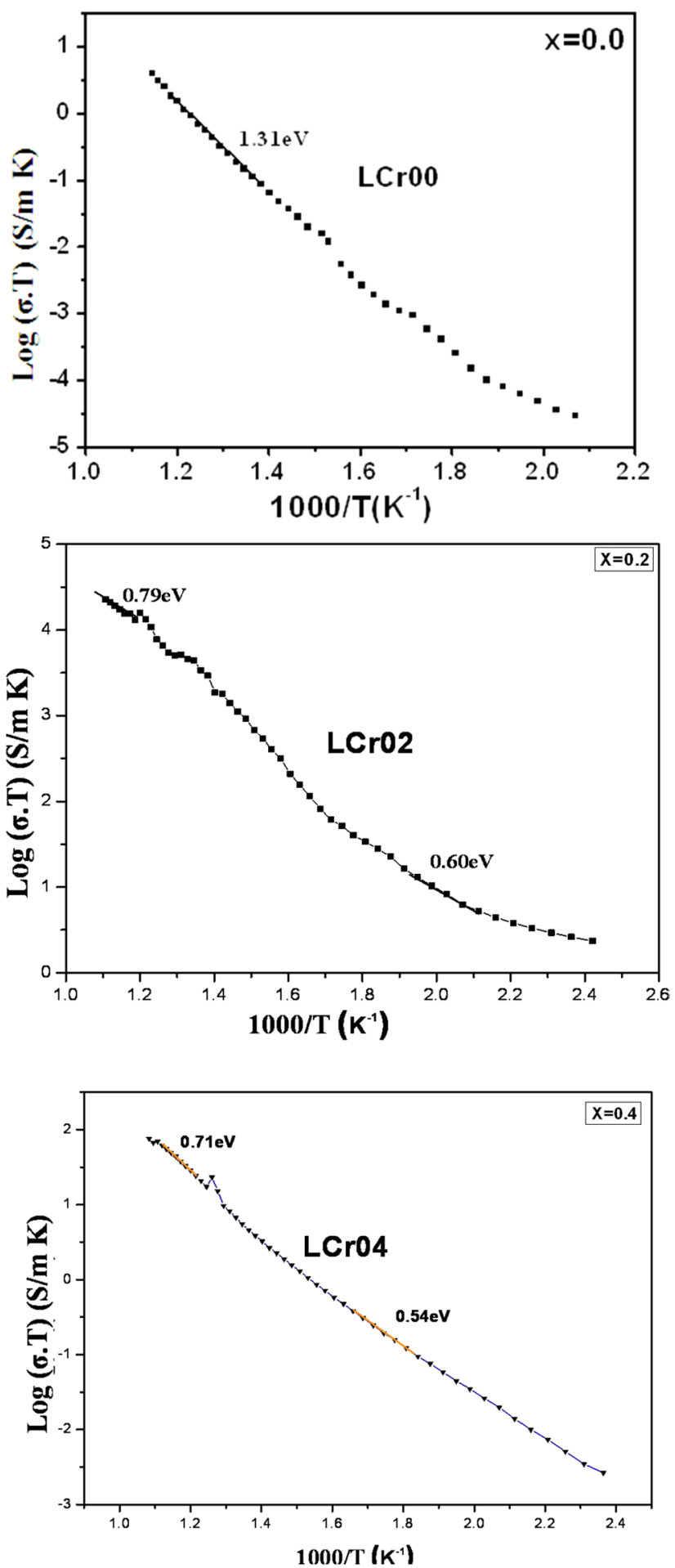

Fig. 6 Arrhenius plots for electrical conductivities of nanocrystalline $\mathrm{Li}_{0.5} \mathrm{Fe}_{2.5-\mathrm{x}} \mathrm{Cr}_{\mathrm{x}} \mathrm{O}_{4}$

grains and grain boundaries wherein grains can perform the high conductivity nature while grain boundaries can show low conductivity behaviour. As far as the normal dielectric behaviour is concerned, with respect to the 

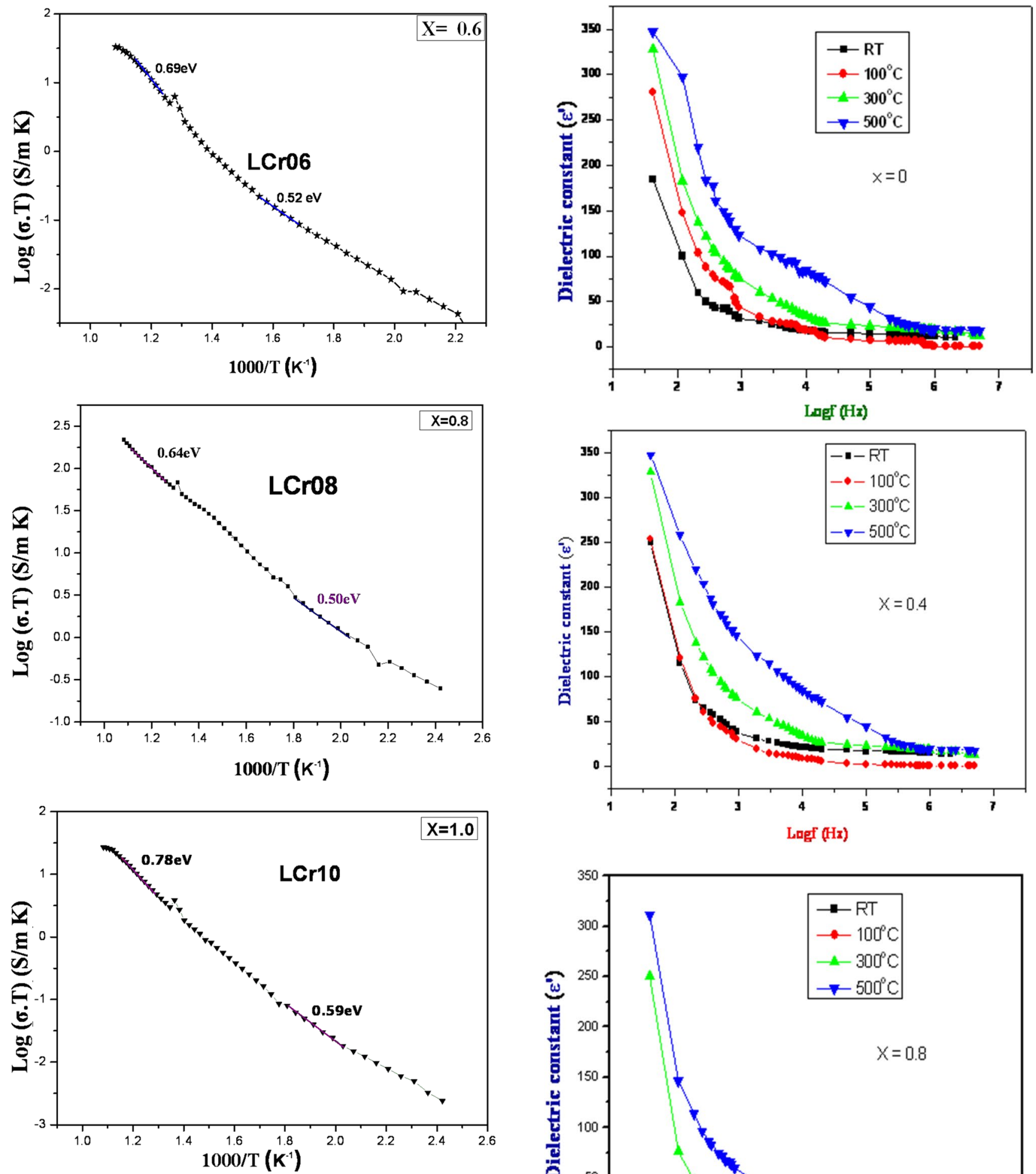

Fig. 6 (continued)

applied frequency the grain boundaries can exhibit more activeness at smaller frequencies and therefore the charge carriers can provide rapid response to the applied frequency which in turn caused to larger amount
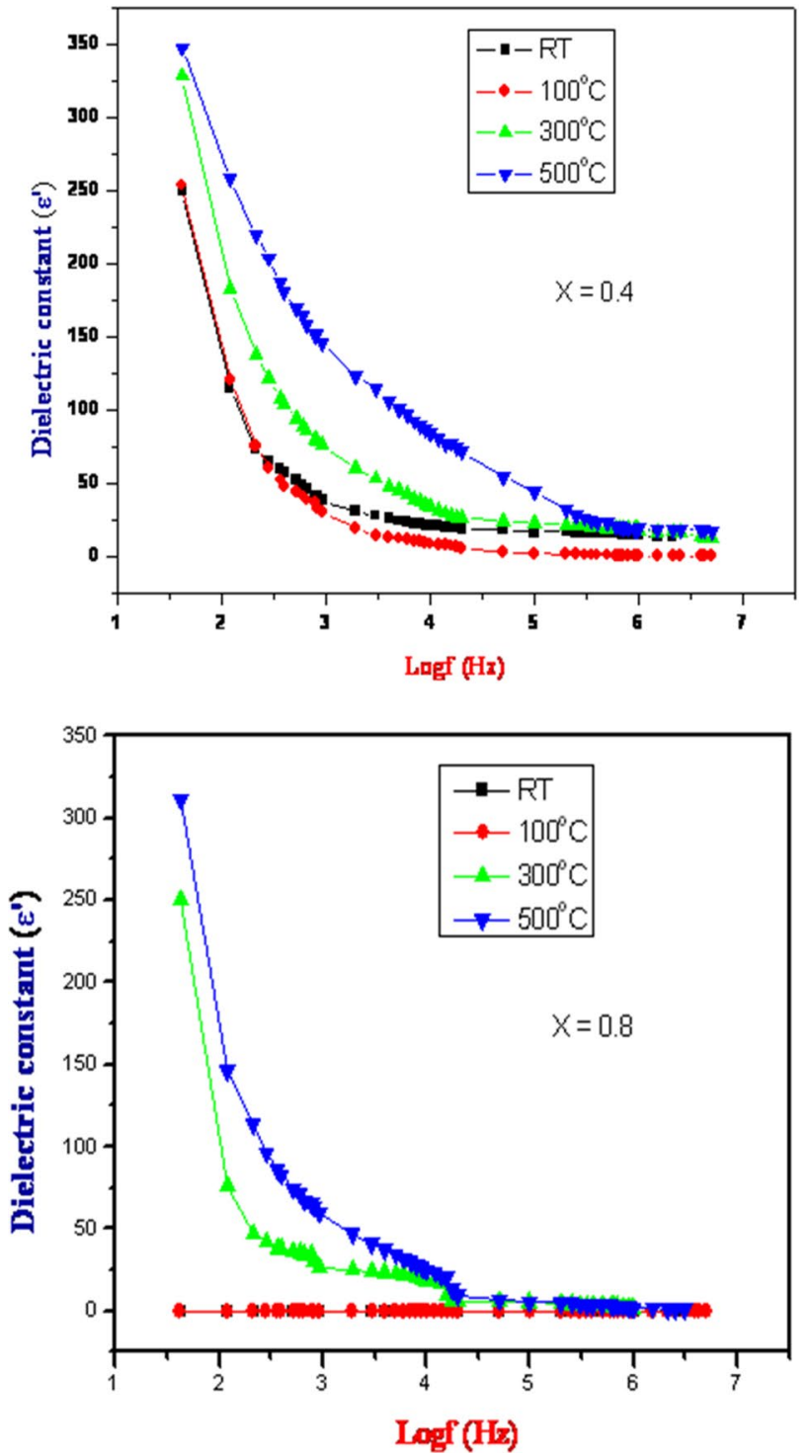

Fig. 7 Frequency dependence of $\varepsilon^{\prime}$ and $\tan \delta$ of $\mathrm{Li}-\mathrm{Cr}$ ferrites at $\mathrm{x}=0.0,0.4,0.8$ and 1.0 

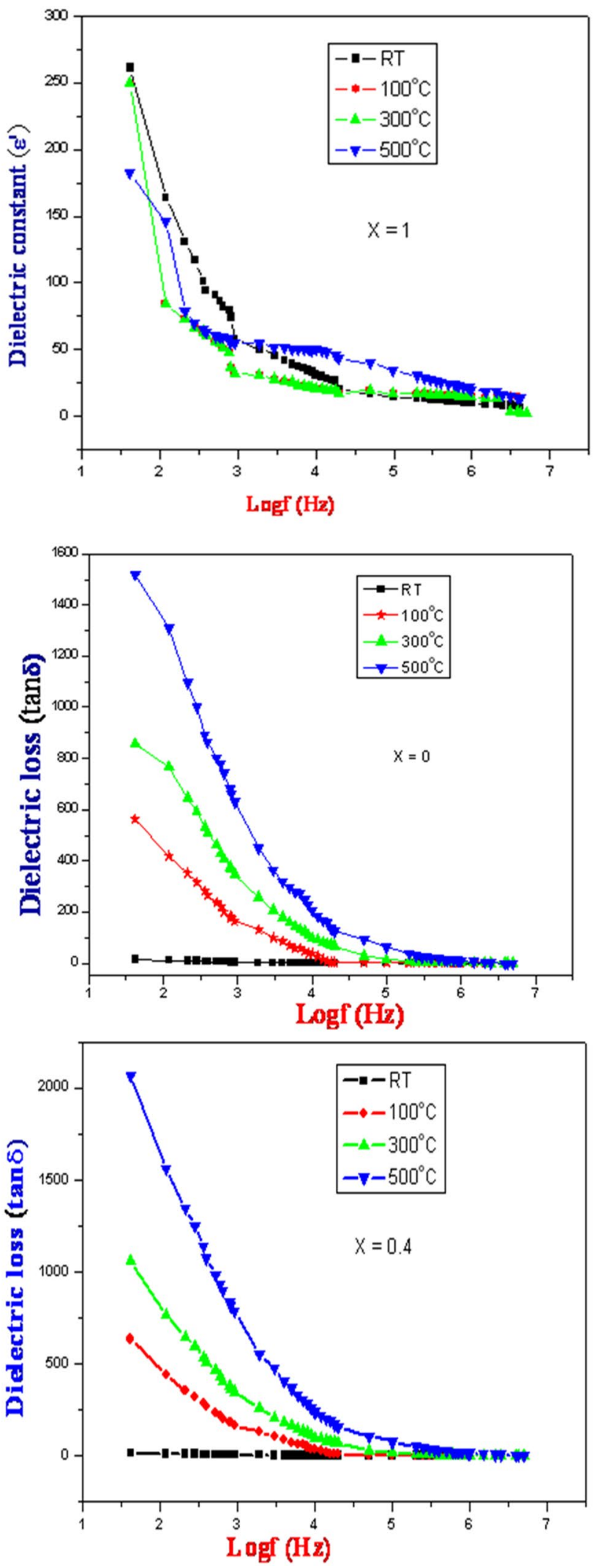

Fig. 7 (continued) of polarization. Thus, charge carriers in bigger number were accumulated at grain boundary interface leading to high $\varepsilon^{\prime}$ and $\tan \delta$. Anyhow, the grains can be more active at high frequencies. Above certain frequencies the electron hopping can't follow the alternating field frequencies. Hence, the reduction in numerical values of $\varepsilon^{\prime}$ and $\tan \delta$ occurred with increase in frequencies. As the temperature was increased from room temperature (RT) to $500{ }^{\circ} \mathrm{C}$, the dielectric constant as well as dielectric loss tangent values were increased. It can be attributed to the increase of polarization with increase of temperature.

The dielectric constant revealed high values for all the materials approximately $300-350$ in magnitude at $100 \mathrm{~Hz}$ frequency. The similar parameter values were noticed to be of order 1-26 at $5 \mathrm{MHz}$. This established a fact that all the materials expressed huge value of dielectric constant at smaller frequencies and low value at larger frequencies. This may be due to its subsequent decay of polarization from high to low at the corresponding applied field frequencies. In addition, at higher frequencies, the constant trend of dielectric constant and loss was mainly adopted owing to the constant Maxwell-Wagner interfacial or space charge polarization. In the same fashion, the dielectric loss values were also reported to be 1600-3000 at $100 \mathrm{~Hz}$ frequency. However, these values (except for $x=1$ ) were not observed at higher frequencies and were approximately noted to be of order 48-93 at high frequencies. Hence, these kinds of high lossy materials can exhibit potential applications as dielectric absorbers where as the materials of high dielectric constant can show applications as charge stored capacitor and microwave devices at high frequencies [33].

The ac-electrical conductivity $\left(\sigma_{\mathrm{ac}}\right)$ was computed using a standard relation mentioned in references [37-42]. In Fig. 8, the frequency dependence of ac-electrical conductivity of present ferrite system was described at room temperature. It was obviously understood from Fig. 8 that the ac-conductivity was almost constant up to $\log f=3$. This can be observed as a result of weak response of the charge carriers at smaller frequencies [43-47]. However, at high frequencies, i.e., above $\log f=3$, a sharp increasing trend of ac-conductivity was noticed. This was occurred owing to the quick response of charge carriers at high frequencies. Thus, huge enhancement of ac- conductivity can be identified for all compositions at high frequencies. Similar observations were observed in the literature [46]. In addition, the exchange of electrons can be become very high at high frequencies $[43,44]$. In comparison, it was also noted that the ac-electrical conductivity was increasing as a function of $\mathrm{Cr}$-content. This type of variation trend was already discussed in the dc-electrical conductivity behavior of present samples with composition. 

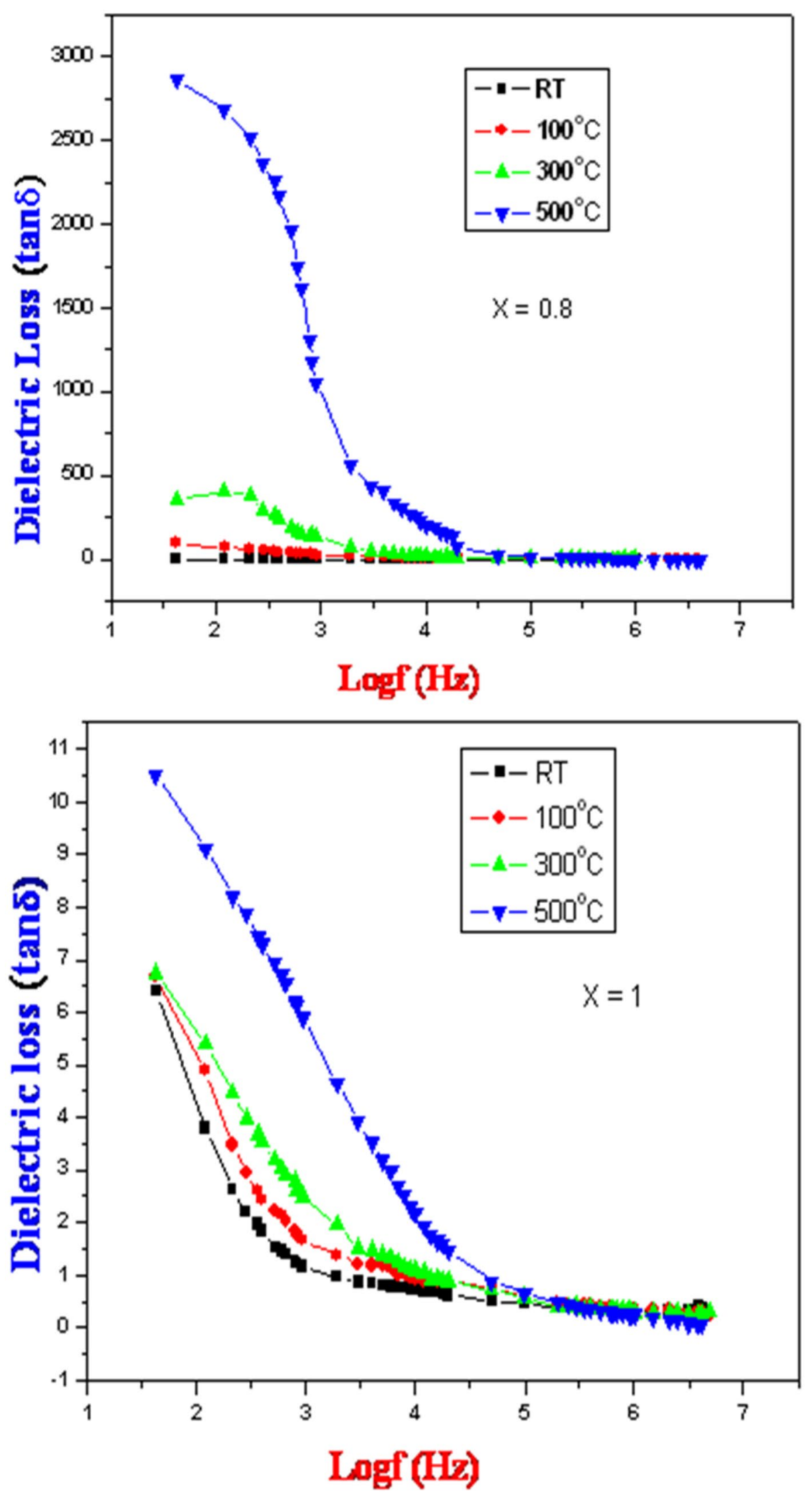

Fig. 7 (continued) 


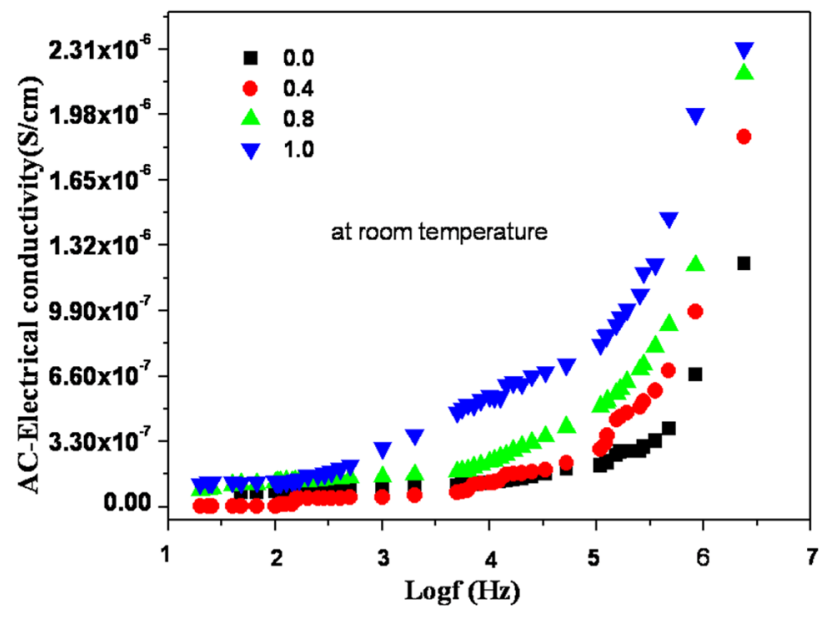

Fig. 8 Frequency dependence of ac-conductivity of $\mathrm{Li}-\mathrm{Cr}$ ferrites at $x=0.0,0.4,0.8 \& 1.0$

\section{Conclusion}

The $\mathrm{Li}-\mathrm{Cr}$ nanoferrites at $\mathrm{x}=0.0,0.4,0.8$ and 1.0 were prepared using the citrate-gel auto combustion technique. The X-ray diffraction patterns confirm the cubic spinel structure formation. The lattice constant and crystallite size values were decreased with increase in $\mathrm{Cr}$-content. The conduction mechanism in ferrites was observed due to the hopping of charge carriers. It was seen that the discontinuity in the log (.) versus $1000 /$ graph showed the Curie point of the prepared ferrite samples. The Curie temperature of the prepared $\mathrm{Li}-\mathrm{Cr}$ ferrites was decreased with the increase of the $\mathrm{Cr}$-concentration. The particles were almost spherical in shape and the average particle size was observed from the TEM. The prepared high lossy materials $(x=0,0.4$ and 0.8$)$ may exhibit potential applications as dielectric absorbers where as the materials of high dielectric constant may show applications as charge stored capacitor and microwave devices at high frequencies.

Acknowledgements The authors are very grateful to Prof. G. Prasad, Head, Department of Physics, University College of Science, Osmania University, Hyderabad for his encouragement. The authors also thankful to UPE-UGC-OU and DST-PURSE-OU.

\section{Compliance with ethical standards}

Conflict of interest The authors declare that we have no conflicts of interest.

Ethical approval The work is contributed by the all authors according to the priority given in the name section. In addition, these samples are not creating any problems to the nature or living organic matter.

\section{References}

1. Schloeman $E$ (2000) Advances in ferrite microwave materials and devices. J Magn Magn Mater 209:15-20

2. Kang S-H, Yoo H-I (1999) Nonstoichiometry $(\delta)$ and high-temperature thermodynamic properties of $\left(\mathrm{Mg}_{0.22} \mathrm{Mn}_{0.07} \mathrm{Fe}_{0.71}\right)_{3-\delta} \mathrm{O}_{4}$ ferrite spinel. J Solid State Chem 145:276-282

3. Abe M, Kuroda J, Matsumoto M (2002) Granular composites containing "micro-onions", permeability, and permittivity calculated for application to microwave absorbers. J Appl Phys 91:7373-7375

4. Verma S, Joy PA (2005) Magnetic properties of superparamagnetic lithium ferrite nanoparticles. J Appl Phys 98:124312-124319

5. Widatallah HM, Johnson C, Berry F, Pekala M (2001) Synthesis, structural, and magnetic characterisation of magnesium-doped lithium ferrite of composition $\mathrm{Li}_{0.5} \mathrm{Fe}_{2.5} \mathrm{O}_{4}$. Solid State Commun 120:171-175

6. Argentina GM, Baba PD (1974) Microwave lithium ferrites: an overview. IEEE Trans Microw Theory Technol 22:652-658

7. Baijal JS, Phanjoubam S, Kothari D, Prakash C, Kishan P (1992) Hyperfine interactions and magnetic studies of $\mathrm{Li}-\mathrm{Mg}$ ferrites. Solid State Commun 83:679-682

8. Fu YP, Hsu CS (2005) $\mathrm{Li}_{0.5} \mathrm{Fe}_{2.5-x} \mathrm{Mn}_{x} \mathrm{O}_{4}$ ferrite sintered from microwave-induced combustion. Solid State Commun 134:201-206

9. Ravinder D, Balachander L, Venudhar YC (2001) Electrical conductivity in manganese-substituted lithium ferrites. Mater Lett 49:267-271

10. Sileo EE, Roteldo R, Jacobo SE (2002) Nickel-Zinc ferrites prepared by the citrate precursor method. J Phys B 320:257-260

11. Bellad SS, Pujar RB, Chougule BK (1998) Structural and magnetic properties of some mixed $\mathrm{Li}-\mathrm{Cd}$ ferrites. Mater Chem Phys 52:166-169

12. Ravinder D (1992) Dielectric behaviour of mixed lithium-zinc ferrites. J Mater Sci Lett 11:1498-1500

13. Yen-Pei $F$ (2006) Microwave-induced combustion synthesis of $\mathrm{Li}_{0.5} \mathrm{Fe}_{2.5-x} \mathrm{Cr}_{x} \mathrm{O}_{4}$ powder and their characterization. Mater Res Bull 41:809-816

14. Krishnan $\mathrm{R}$ (1977) Magnetic anisotropy in $\mathrm{Li}_{0.5} \mathrm{Fe}_{2.5-x} \mathrm{Cr}_{x} \mathrm{O}_{4}$ crystals. Physica B+C 86-88:1457-1458

15. Slawska-Waniewska A, Krishnan $R$ (1980) Pressure dependence of anisotropy constants $\mathrm{K}_{1}, \mathrm{~K}_{2}$ in $\mathrm{Li}_{0.5} \mathrm{Fe}_{2.5-x} \mathrm{Cr}_{x} \mathrm{O}_{4}$. J Magn Magn Mater 15-18:579-581

16. Fernandez-Barquın L, Kuznetsov MV, Morozov YG, Pankhurst QA, Parkin IP (1999) Group 4 metal complexes that contain a thioether-functionalized phenolato ligand: synthesis, structure, and 1-hexene polymerization. Int J Inorgan Mater 1:311-316

17. Sunandana CS, Phaninath D (1986) Photoacoustic spectra of $\mathrm{Fe}^{3+}$ and $\mathrm{Cr}^{3+}$ in lithium chromo-ferrites, $\mathrm{Li}_{0.5} \mathrm{Fe}_{2.5-x} \mathrm{Cr}_{x} \mathrm{O}_{4}$ $(0 \leq x \leq 2.5)$. Solid State Commun 58:115-119

18. Srivastava M, Layek S, Singh J, Das AK, Verma HC, Ojha AK, Kim $\mathrm{NH}$, Lee JH (2014) Synthesis, magnetic and Mössbauer spectroscopic studies of $\mathrm{Cr}$ doped lithium ferrite nanoparticles. J Alloys Compd 591:174-180

19. Srivastava M, Mishra RK, Singh J, Srivastava N, Kim NH, Lee JH (2015) Consequence of $\mathrm{pH}$ variation on the dielectric properties of $\mathrm{Cr}$-doped lithium ferrite nanoparticles synthesized by the sol-gel method. J Alloys Compd 645:171-177

20. Yen-Pei F, Hung D-S, Yao Y-D (2009) Microwave properties of chromium-substituted lithium ferrite. Ceram Int 35:2179-2184

21. Yen-Pei F, Wen S-B, Yen C-C (2009) Magnetic and thermal expansion properties of chromium-substituted lithium ferrite. Ceram Int 35:943-947

22. Mane DR, Patil $S$, Birajdar DD, Kadam AB, Shirsath $\mathrm{SE}$, Kadam RH (2011) Sol-gel synthesis of $\mathrm{Cr}^{3+}$ substituted $\mathrm{Li}_{0.5} \mathrm{Fe}_{2.5} \mathrm{O}_{4}$ : cation 
distribution, structural and magnetic properties. Mater Chem Phys 126:755-760

23. Yen-Pei Fu (2009) Electrical conductivity and magnetic properties of $\mathrm{Li}_{0.5} \mathrm{Fe}_{2.5-x} \mathrm{Cr}_{x} \mathrm{O}_{4}$ ferrite. Mater Chem Phys 115:334-338

24. West RG, Blankenship AC (1967) Magnetic properties of dense lithium ferrites. J Am Ceram Soc 50:343-349

25. Jauhar S, Goyal A, Lakshmi N, Chandra K, Singhal S (2013) Doping effect of $\mathrm{Cr}^{3+}$ ions on the structural, magnetic and electrical properties of $\mathrm{Co}-\mathrm{Cd}$ ferrites: a study on the redistribution of cations in $\mathrm{CoCd}_{0.4} \mathrm{Cr}_{x} \mathrm{Fe}_{1.6-x} \mathrm{O}_{4}(0.1 \leq x \leq 0.6)$ ferrites. Mater Chem Phys 139:836-843

26. Kumar NS, Suvarna RP, Naidu KCB (2019) Phase change and ferroelectric nature of microwave heated lead cobalt titanate nanoparticles prepared by sol-gel method. Int J Appl Ceram Technol 16:130-137

27. Kumar NS, Suvarna RP, Naidu KCB (2018) Structural and ferroelectric properties of microwave heated lead cobalt titanate nanoparticles synthesized by sol-gel technique. J Mater Sci Mater Electron 29:4738-4742

28. Kothandan D, Kumar RJ, Prakash M, Naidu KCB (2018) Structural, morphological and optical properties of $\mathrm{Ba}_{1-\mathrm{x}} \mathrm{Cu}_{\mathrm{x}} \mathrm{TiO}_{3}$ $(x=0.2,0.4,0.6,0.8)$ nanoparticles synthesized by hydrothermal method. Mater Chem Phys 215:310-315

29. Kumar NS, Suvarna RP, Naidu KCB, Kumar GR, Ramesh S (2018) Structural and functional properties of sol-gel synthesized and microwave heated $\mathrm{Pb}_{0.8} \mathrm{CO}_{0.2-z} \mathrm{La}_{\mathrm{z}} \mathrm{TiO}_{3}(\mathrm{z}=0.05-0.2)$ nanoparticles. Ceram Int 44:19408-19420

30. Ramaprasad T, Kumar RJ, Naresh U, Prakash M, Kothandan D, Naidu KCB (2018) Effect of $\mathrm{pH}$ value on structural and magnetic properties of $\mathrm{CuFe}_{2} \mathrm{O}_{4}$ nanoparticles synthesized by low temperature hydrothermal technique. Mater Res Express 5:095025. https://doi.org/10.1088/2053-1591/aad860

31. Naidu KCB, Suresh Kumar G, Ranjith Kumar N et al (2018) Temperature and frequency dependence of complex impedance parameters of microwave sintered NiMg ferrites. J Aust Ceram Soc 1:1. https://doi.org/10.1007/s41779-018-0260-x

32. Naidu KCB, Reddy VN, Sarmash TS, Kothandan D, Subbarao T, Kumar NS (2019) Structural, morphological, electrical, impedance and ferroelectric properties of $\mathrm{BaO}-\mathrm{ZnO}-\mathrm{TiO}_{2}$ ternary system. J Aust Ceram Soc 55:201-218

33. Kumar DS, Naidu KCB, Rafi MM, Nazeer KP, Begam AA, Kumar GR (2018) Structural and dielectric properties of superparamagnetic iron oxide nanoparticles (SPIONs) stabilized by sugar solutions. Mater Sci Pol 36:123-133

34. Sivakumar D, Naidu KCB, Nazeer KP, Rafi MM, Rameshkumar G, Sathyaseelan B, Killivalavan G, Begam AA (2018) Structural characterization and dielectric properties of superparamagnetic iron oxide nanoparticles. J Korean Ceram Soc 55:230-238

35. Kumar NS, Suvarna RP, Naidu KCB (2018) Sol-gel synthesized and microwave heated $\mathrm{Pb}_{0.8-y} \mathrm{La}_{\mathrm{y}} \mathrm{CO}_{0.2} \mathrm{TiO}_{3}(\mathrm{y}=0.2-0.8)$ nanoparticles: structural, morphological and dielectric properties. Ceram Int 44:18189-18199
36. Zangina T, Hassan J, Azis RS et al (2018) Structural, electrical conductivity and dielectric relaxation behavior of $\mathrm{LiHf}_{2}\left(\mathrm{PO}_{4}\right)_{3}$ ceramic powders. J Aust Ceram Soc 54:307-316

37. Raghuram N, Rao TS, Naidu KCB (2019) Investigations on functional properties of hydrothermally synthesized $\mathrm{Ba}_{1-x} \mathrm{Sr}_{x} \mathrm{Fe}_{12} \mathrm{O}_{19}$ $(x=0.0-0.8)$ nanoparticles. Mater Sci Semicond Process $94: 136-150$

38. Ell MA, Hiti AC (1996) Electrical conductivity of Ni-Mg ferrites. J Phys D Appl Phys 29:501-505

39. Naidu KCB, Padma GT, Rao TS, Kumar NS, Raghuram N (2018) Phase formation and electrical properties of zinc titanate ceramics. Adv Mater 2:37-48

40. Naidu KCB, Madhuri W (2018) Ceramic nanoparticle synthesis at lower temperatures for LTCC and MMIC technology. IEEE Trans Magn. https://doi.org/10.1109/tmag.2018.2855663

41. Kumar NS, Suvarna RP, Naidu KCB (2019) Grain and grain boundary conduction mechanism in sol-gel synthesized and microwave heated $\mathrm{Pb}_{0.8-y} \mathrm{La}_{\mathrm{y}} \mathrm{Co}_{0.2} \mathrm{TiO}_{3}(\mathrm{y}=0.2-0.8)$ nanofibers. Mater Chem Phys 223:241-248

42. Joshi S, Kumar M (2018) Effect of $\mathrm{Ni}^{2+}$ substitution on structural, magnetic, dielectric and optical properties of mixed spinel $\mathrm{CoFe}_{2} \mathrm{O}_{4}$ nanoparticles. Ceram Int 42:18154-18165

43. Naresh U, Kumar RJ, Naidu KCB (2019) Optical, magnetic and ferroelectric properties of $\mathrm{Ba}_{0.2} \mathrm{Cu}_{0.8-x} \mathrm{La}_{\mathrm{x}} \mathrm{Fe}_{2} \mathrm{O}_{4}(\mathrm{x}=0.2-0.6)$ nanoparticles. Ceram Int 1:2. https://doi.org/10.1016/j.ceram int.2019.01.044

44. Boda N, Boda G, Naidu KCB, Srinivas M, Batoo KM, Ravinder D, Reddy AP (2019) Effect of rare earth elements on low temperature magnetic properties of $\mathrm{Ni}$ and Co-ferrite nanoparticles. J Magn Magn Mater 473:228-235

45. Kumar GR, Basha DB, Naidu KCB, Ramesh S, Srinivas K (2018) Spectroscopic properties of $\mathrm{NiO}, \mathrm{PbO}, \mathrm{CaO}$ and $\mathrm{MgO}$ ionic crystals synthesized by ball milling method. Recent Pat Mater Sci 11:97-107. https://doi.org/10.2174/18744648126661811220 93655

46. Padma GT, Rao TS, Naidu KCB (2019) Preparation, characterization and dielectric properties of sodium alginate/titanium dioxide composite membranes. SN Appl Sci 1:75. https://doi. org/10.1007/s42452-018-0083-y

47. Kumar NS, Suvarna RP, Naidu KCB (2019) Multiferroic nature of microwave-processed and sol-gel synthesized nano $\mathrm{pb}_{1-\mathrm{x}} \mathrm{Co}_{\mathrm{x}} \mathrm{TiO}_{3}(\mathrm{x}=0.2-0.8)$ ceramics. Cryst Res Technol. https:// doi.org/10.1002/crat.201800139

Publisher's Note Springer Nature remains neutral with regard to jurisdictional claims in published maps and institutional affiliations. 